\title{
Effect of Solvent on Concentration Dependence of Hydrodynamic Volumes and GPC Elution Volumes
}

\author{
Hadi Kh. MAHABADI* and Alfred RUdiN \\ Guelph-Waterloo Centre for Graduate Work in Chemistry, \\ University of Waterloo, \\ Waterloo, Ontario, Canada N2L $3 G 1$
}

(Received February 21, 1978)

\begin{abstract}
The models of Yamakawa and Rudin were used to predict the effects of polymer concentration on hydrodynamic volumes of solvated macromolecules and on peak elution volumes in gel-permeation chromatographic (GPC) analyses. Both theories predict GPC behavior accurately for all systems of practical analytical interest. Yamakawa theory calculations predict that a concentration region may exist in which the polymer hydrodynamic volume may be greater in a poorer solvent than in a thermodynamically better solvent. This "cross-over" is noticeable for high molecular weight polymers at relatively high concentrations which are beyond the dilute region for which the theory was developed. Some GPC observations with porous glass packings are nevertheless in qualitative agreement with this prediction. Both models account for the effects of concentration, solvent choice, and polymer molecular weight on GPC column behavior.

KEY WORDS Hydrodynamic Volume / Elution Volume / GPC / Gyration Radius /
\end{abstract}

It is well known that the peak elution volume tends to increase with increasing concentration of a given polymer in the feed to GPC column sets. ${ }^{1-10}$ The concentration effect is more pronounced with polymers of narrow molecular weight distribution and higher mean molecular weight. The effect of concentration decreases as the thermodynamic quality of the GPC solvent deteriorates and is practically non-existent in theta mixtures.

The concentration effect in GPC was explained as a consequence of the reduction of the effective hydrodynamic volume of solvated polymer coils with increasing concentration. A semi-empirical model has been discussed which can be used to correct for concentration effects in GPC. ${ }^{11}$ This model has also been applied to universal calibration in GPC. ${ }^{12}$ The theory did not fit experimental observations in theta solvents and a revision (herein-after referred to as the Rudin model) has been published which is less empirical and which appears to fit the elution behavior of polymers in all

* On leave from Chemical Engineering Department, Arya-Mehr University of Technology, Tehran, Iran.
GPC solvents. ${ }^{9,13-16}$

The Rudin model seems to be the only one which has been used quantitatively to correct GPC data for concentration effects. It was of interest, therefore, to determine whether a model which is accepted as more "orthodox" in current polymer solution theory would also be applicable to this practical problem. In this article, we compare the Rudin model $^{13}$ with Yamakawa's ${ }^{17}$ theory relating the concentration and effective hydrodynamic volume of solvated polymers. It will be shown that the two theories yield equivalent predictions under almost all conditions which are likely to be encountered in GPC analyses. The two deviate only in the case of very high molecular weight polymers at relatively high concentrations in very good solvents. The Rudin model is more convenient to use. The Yamakawa theory predicts that the dimensions of a high molecular weight polymer coil in a good solvent may be smaller than those in a poorer solvent at a given high concentration. The reality of this "cross-over" phenomenon depends on the concentration range 
in which the Yamakawa theory is applicable and this is not clear at present. Some data from GPC separations on porous glass columns are, however, qualitatively consistent with this surprising prediction. Either theory may be used to correct for concentration affects and to serve as a basis for universal calibration in GPC.

\section{Theory}

The hydrodynamic volume, $V_{\mathrm{h}}$, of a solvated polymer molecule is defined as

$$
V_{\mathrm{h}}=v \alpha^{3}
$$

-where $v$ is the unswollen volume and $\alpha$ is the linear expansion coefficient which reflects polymersolvent interactions. ${ }^{18}$ The unswollen volume is that under theta conditions and is given by

$$
v=4 \pi[\eta]_{\theta} M / 3 \phi^{\prime}
$$

in $\mathrm{cm}^{3} /$ molecule, where $\phi^{\prime}$ is the Flory universal constant. This value is proposed as $3.1 \times 10^{24}$ with the units of $[\eta]$ in $\mathrm{cm}^{3} / \mathrm{g}$. Use of a solventdependent value of $\phi^{\prime}$ is not necessary in this context because molecular dimensions calculated with the aid of Flory's value are within experimental uncertainty of the available data in all cases. The term $[\eta]_{\theta}$ in eq. 2 is the intrinsic viscosity of the polymer in a theta solvent and is given by

$$
[\eta]_{\theta}=K_{\theta} M^{0.5}
$$

Here $K_{\theta}$ is the Mark-Houwink constant of the polymer under theta conditions. It is evident also from eq 3 and its predecessors that consideration is restricted to a polymer sample which is monodisperse in molecular weight. $K_{\theta}$ is assumed to be a constant which can be calculated with sufficient accuracy from the Flory-Fox ${ }^{19}$ equation:

$$
K_{\theta}=2.5 \times 10^{-1} \frac{\beta^{3}}{M_{0}^{3 / 2}}\left(\text { in } \mathrm{cm}^{3} / \mathrm{g}\right)
$$

In eq $4, \beta$ (in $\AA$ ) is an effective bond length typically equal to triple the carbon-carbon bond distance $(\beta=4.96 \AA \text { for polystyrene })^{20}$ and $M_{0}$ is half the formula weight of the repeating unit in a vinyl polymer. For polystyrene, eq 4 yields $K_{\theta}=$ $8.135 \times 10^{-2} \mathrm{~cm}^{3} / \mathrm{g}$, compared to a measured value of $7.023 \times 10^{-2} \mathrm{~cm}^{3} / \mathrm{g}^{21,22}$

In any solvent the intrinsic viscosity of the particular polymer is given by the Mark-Houwink equation with appropriate constants

$$
[\eta]=K M^{a^{\prime}}
$$

The radius of gyration of the solvated polymer at infinite dilution, $S_{0}$, follows from

$$
S_{0}=\left([\eta] M / \phi^{\prime}\right)^{1 / 3}
$$

The linear expansion factor can be related to the intrinsic viscosity by ${ }^{19}$

$$
\alpha_{\eta}=\left([\eta] /[\eta]_{\theta}\right)^{1 / 3}
$$

where $\alpha_{\eta}$ is a corresponding factor pertaining to viscosity measurements. As a useful approximation

$$
\alpha_{0} \simeq \alpha_{\eta}=\left(K / K_{\theta}\right)^{1 / 3} M^{a^{\prime}-0.5 / 3}
$$

where $\alpha_{0}$ is the expansion coefficient at infinite dilution.

The concentration dependence of $V_{\mathrm{h}}$ (eq 1$)$ can be obtained through the relation between $\alpha$ and concentration. There have been several attempts to calculate this relation. ${ }^{17,23-26}$ The perturbation theory of Yamakawa, which is of particular interest here, predicts that $\alpha$ decreases with increasing concentration, $c$, according to ${ }^{17}$

$$
\alpha=\alpha_{0} \exp \left[-0.345 Z \phi_{2}\left(\xi_{0}\right)[\eta]_{\theta} c\right]
$$

where

$$
\phi_{2}\left(\xi_{0}\right)=1-\left\{\left[1-\exp \left(-5.731 \xi_{0}\right)\right] / 5.731 \xi_{0}\right\}
$$

Here $\xi_{0}$ is a parameter given by $\xi_{0}=Z / \alpha_{0}{ }^{3}$ and $Z$ is the so-called interaction parameter, which is related to $\alpha_{0}$ by

$$
\alpha_{0}^{2}=0.541+0.459(1+6.04 Z)^{0.46}
$$

It is evident from eq 11 that the limiting value of $Z$ is zero as the polymer solution approaches theta conditions.

Introduction of eq 2, 7, and 9 and the value of $\phi^{\prime}$ into eq 1 yields

$V_{\mathrm{h}}=(4 \pi / 9.3) \times 10^{-24} M[\eta] \exp \left(-1.035 Z \phi_{2}\left(\xi_{0}\right)[\eta]_{\theta} c\right)$

with $c$ in $\mathrm{g} / \mathrm{m} l$ and $[\eta]$ in $\mathrm{m} l / \mathrm{g}$. Equation 12 predicts the concentration dependence of $V_{\mathrm{h}}$, with the parameters defined by eq 8,10 , and 11 .

Rudin's model ${ }^{13}$ results in an expression of the form

$$
V_{\mathrm{h}}=\frac{4 \pi[\eta] M}{\left.\left.9.3 \times 10^{24}+4 \pi N_{0} c(] \eta\right]-[\eta]_{\theta}\right)}
$$

where $N_{0}$ is Avogadro's constant. Equations 12 and 13 are similar in that they define identical effective hydrodynamic volumes in the limits of infinite dilution and at a higher, critical concentration, $c_{x}$, when the volume has shrunk to its theta condition value. Yamakawa's theory predicts 
an exponential decrease of the hydrodynamic volume between these limits. In Rudin's model the reciprocal of the hydrodynamic volume is assumed to increase linearly with concentration between zero and the critical value of this parameter. This is equivalent in effect to a truncated series expansion of the logarithmic concentration term. That is to say, Rudin's model employs a term $\left(1+f_{1}(c)\right)^{-1}$ instead of Yamakawa's $\exp \left(-f_{2}(c)\right)$. Both approaches yield equivalent results in most cases of interest.

Complete specification of eq 12 requires knowledge of the constants $K_{\theta}, K$, and $a^{\prime}$ and the polymer molecular weight, $M$. Equation 8 first yields $\alpha_{0}$ and insertion of this value into eq 11 permits evaluation of the interaction parameter $Z$. The value of $\phi_{2}$ can then be estimated by using the calculated $\alpha_{0}$ and $Z$ in eq 10. Finally, insertion of $Z$ and $\phi_{2}$ into eq 12 produces an estimate of $V_{\mathrm{h}}$ at a given concentration $c$. These calculations are more laborious than those involved in use of eq 13.

The dependence of $Z$ and $\phi_{2}$ on $\alpha_{0}$ are reported in the literature. Both relations are shown here in Figure 1, for the sake of clarity. Both $\phi_{2}$ and $Z$ approach zero as $\alpha_{0}$ is reduced toward its theta condition value of unity. It is clear from eq 12 and 13 that the effective hydrodynamic volume decreases with increasing concentration and that the extent of this decrease is greater the larger the dimensions of the solvated polymer at infinite dilution. That is to say, concentration effects are more pronounced with higher molecular polymers and in good solvents. ${ }^{11}$

The critical concentration $c_{x}$, which is defined as the concentration at which the dimensions of the solvated macromolecules have been reduced to their theta condition values, can be estimated by substituting $\alpha=1$ into eq 9

$$
c_{x}=\frac{\ln \alpha_{0}}{0.345 Z \phi_{2}[\eta]_{\theta}}
$$

The corresponding relation derived from other considerations in Rudin's mode ${ }^{13}$ is

$$
c_{x}=\frac{3 \phi^{\prime}}{4 \pi N_{0}[\eta]_{\theta}}
$$

While eq 15 predicts that $c_{x}$ is independent of the kind of solvent, the prediction of eq 14 is that $c_{x}$ varies with the solvent type. For a given mole-

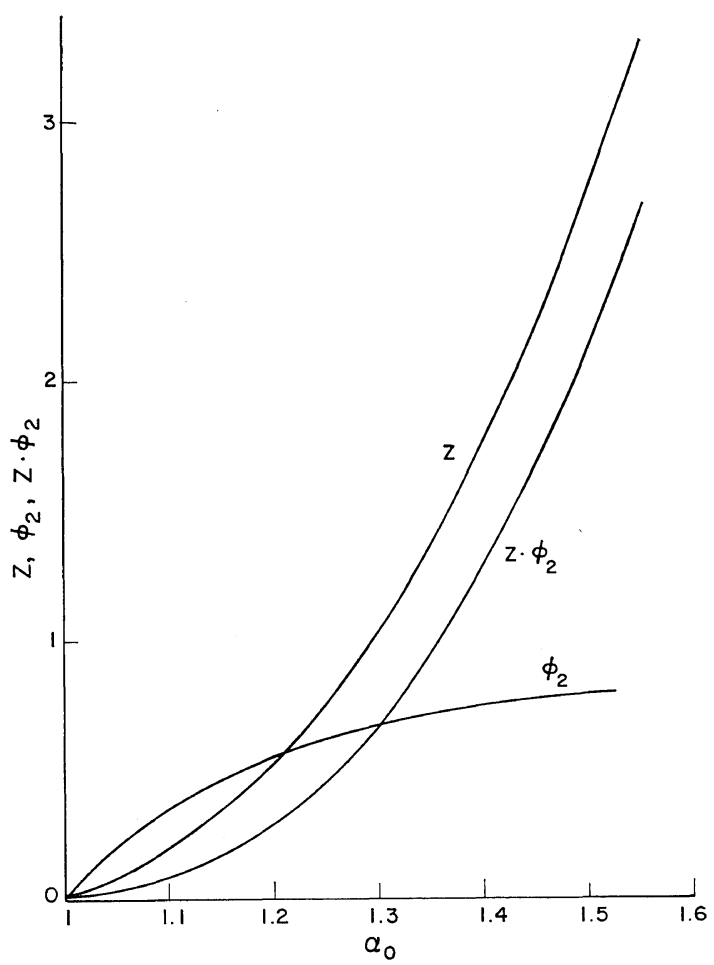

Figure 1. Relations between $\alpha_{0}$ (eq 8), $Z$ (eq 11), and $\phi_{2}$ (eq. 10).

cular weight of polymer, $c_{x}$ in a good solvent is less than that in a poor solvent and is infinite in a theta solvent. However eq 14 and 15 yield similar results for intermediate regions of polymer dimensions. In both models the dimensions of the solvated molecules are assumed to decrease to those prevailing under theta conditions and to remain at their theta condition values for all $c \geq c_{x}$.

\section{RESULTS}

\section{Concentration Dependence of the Radius of Gyration}

Rudin's model has been shown ${ }^{13}$ to predict the results of direct measurements of the radius of gyration of a polystyrene molecule in toluene. ${ }^{27}$ Similar calculations are reported here for the Yamakawa model. The original authors ${ }^{27}$ have computed radii of gyration from the models of Yamakawa, ${ }^{17}$ Grimley,${ }^{28}$ Fixman,${ }^{25}$ and Simha and Zakin, ${ }^{29}$ which do not agree very closely with the measured values. Our calculations, however, show a good coincidence of experimental figures 
Table I. Parameters used in the calculation of hydrodynamic volumes

\begin{tabular}{|c|c|c|c|c|c|c|}
\hline Polymer & Solvent & $\underset{{ }^{\circ} \mathrm{C}}{\text { Temp }}$ & $\begin{array}{l}K_{\theta} \times 10^{3}, \\
\mathrm{~m} l / \mathrm{g}^{21,22}\end{array}$ & $\begin{array}{c}K \times 10^{3}, \\
\mathrm{~m} l / \mathrm{g}\end{array}$ & $a^{\prime}$ & Ref \\
\hline Polystyrene & Tetrahydrofuran & 25 & 72.03 & 6.82 & 0.766 & 4 \\
\hline Polystyrene & Butanone & 25 & 72.03 & 19.5 & 0.635 & 21 \\
\hline Polystyrene & Chloroform & 30 & 77.0 & 4.9 & 0.794 & 8 \\
\hline Polystyrene & Benzene & 25 & 72.03 & 9.18 & 0.743 & 34 \\
\hline $\begin{array}{l}\text { Poly(dimethyl- } \\
\text { siloxane) }\end{array}$ & Chloroform & 30 & 74 & 5.4 & 0.77 & 35 \\
\hline Polystyrene & Toluene & 25 & 72.03 & 17.0 & 0.69 & 30 \\
\hline
\end{tabular}

with those from Yamakawa's theory.

In this case, eq 6 is used at the theta condition along with eq 7 and 9 . The combined expression can be simplified to

$$
S=\left(\frac{[\eta] M}{3.1 \times 10^{24}}\right)^{1 / 3} \exp \left(-0.345 Z \phi_{2}\left(\xi_{0}\right)[\eta]_{\theta} c\right)
$$

with $S$ in $\mathrm{cm},[\eta]$ in $\mathrm{cm}^{3} \mathrm{~g}^{-1}$, and $c$ in $\mathrm{g} \mathrm{cm}^{-3}$. For these calculations, $K_{\theta}$ (eq 4 ) was given its experimental value of $7.023 \times 10^{-2} \mathrm{~cm}^{3} \mathrm{~g}^{-1} \cdot{ }^{21,22}$ This and other parameters used in the present hydrodynamic volume calculations are listed in Table I.

Table II compares experimental values of the radius of gyration with those calculated from eq 16

Table II. Radius of gyration $(S)$ of polystyrene $(M=110,000)$ in toluene at $25^{\circ} \mathrm{C}$

\begin{tabular}{cccl}
\hline \multirow{2}{*}{$\begin{array}{c}\text { Concentration, } \\
c, \text { gcm }^{-3}\end{array}$} & \multicolumn{3}{c}{$S, \AA$} \\
\cline { 2 - 4 } & $\begin{array}{c}\text { Calcd } \\
\text { (eq 16) }\end{array}$ & $\begin{array}{c}\text { Calcd } \\
(\text { eq 17) }\end{array}$ & \multicolumn{1}{c}{ Exptl } \\
\hline 0 & 122 & 122 & $119^{31} 120^{32}$ \\
0.005 & 119 & 118 & $119^{27}$ \\
0.031 & 104 & 104 & $101.5^{27}$ \\
0.061 & 95 & 95 & $96.5^{27} 91 \pm 5^{33}$ \\
\hline
\end{tabular}

(Yamakawa) and eq 17 (Rudin). The latter derives in an obvious manner from eq 13:

$$
S=\left[\frac{3[\eta] M}{9.3 \times 10^{24}+4 \pi N_{0} c\left([\eta]-[\eta]_{\theta}\right)}\right]^{1 / 3}
$$

It should be noted that $c_{x}$ for this system is 0.048 $\mathrm{g} \mathrm{cm}^{-3}$ from eq 14 or $0.046 \mathrm{~g} \mathrm{~cm}^{-3}$ from eq 15 , and the calculated value of $S$ at $c=0.061 \mathrm{~g} \mathrm{~cm}^{-3}$ is therefore that for theta conditions.

This comparison is limited by the number of experimental data points available. It is evident that the two models are not distinguishable in this system and both accord with experiment within the reliability of the latter values.
Concentration Dependence of GPC Elution Volumes

The elution volume in gel-permeation analysis of polymers depends on sample concentration as well as on polymer molecular weight. The elution volume observed is a function of the logarithm of the hydrodynamic volume of the solvated polymer species. The hydrodynamic volume of a given species is, in turn, inversely related to the concentration and the elution volume-molecular weight relation is therefore not uniquely determined.

The Rudin model ${ }^{13}$ and an earlier, semiempirical version ${ }^{11}$ account for the influence of concentration on the effective volume of solvated polymer molecules and hence correct for concentration effects in gel permeation chromatography (GPC). The Yamakawa and Rudin models are compared here in this context.

The connection between eq 12 or eq 13 and the universal calibration curve of $\log M[\eta]$ against $V_{\mathrm{e}}$ (elution volume) is straightforward. The Einstein equation for the viscosity, $\eta$, of a dilute suspension of rigid spheres in a medium of viscosity $\eta_{0}$ is

$$
\eta=\eta_{0}(1+2.5 F)
$$

where $F$ is the volume fraction of suspended material and is equal, in general, to

$$
F=\left(N_{0} c V_{\mathrm{h}} / M\right)
$$

In the limit of infinite dilution, eq 5, 18, and 19 are equivalent to

$$
\lim _{c \rightarrow 0} \frac{1}{c}\left[\frac{\eta}{\eta_{0}}-1\right] M=[\eta] M=2.5 N_{0} V_{\mathrm{h}}
$$

Since the hydrodynamic volume of the solute seems to be the appropriate universal calibration procedure for the GPC technique ${ }^{36-38}$ it is not surprising that $\ln [\eta] M\left(=\right.$ constant $\left.+\ln V_{\mathrm{h}}\right)$ frequently produces a common plot for different 
fractionated polymers in a given GPC solvent. ${ }^{12,39,40}$

It is clear, however, from eq 12 and 13 that the equivalence of $\ln M[\eta]$ and $\ln V_{\mathrm{h}}$ holds only for infinitely dilute solutions of polymers or for any concentration of a polymer in a theta solvent. In other cases, the plots of $\ln M[\eta] v s$. $V_{\mathrm{e}}$ will be shifted to higher $V_{\mathrm{e}}$ than that of $\ln V_{\mathrm{h}}$ against $V_{\mathrm{e}}$, because $V_{\mathrm{h}}$ is actually somewhat smaller than that which can be calculated at infinite dilution.

It is necessary to establish an effective polymer concentration for subsequent calculations, since the hydrodynamic volume of a solvated polymer will depend on concentration. Fortunately, previous studies ${ }^{11-13}$ have shown that the Rudin model yields a common log (hydrodynamic volume)-elution volume curve for all concentrations and molecular weights of a fractionated polymer with estimates of hydrodynamic volume based on the nominal concentration of the solute in the GPC column set inlet. Infinite dilution hydrodynamic volumes of whole polymers calculated

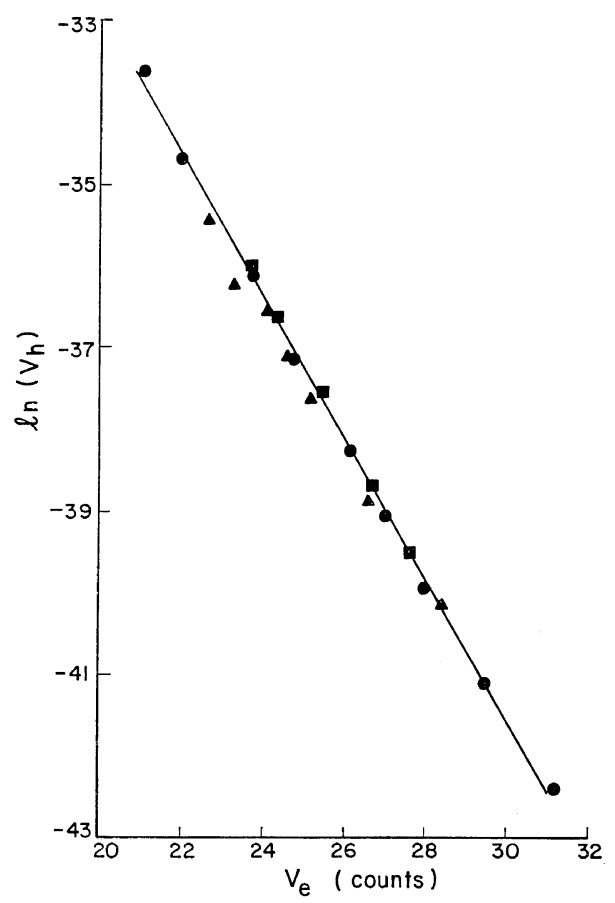

Figure 2. In (hydrodynamic volume, $V_{\mathrm{h}}$ ) vs. elution volume, $V_{\mathrm{e}}$, for polystyrene (-), poly(dimethylsiloxane) " $\mathrm{D}$ " fractions (圄), and poly(dimethylsiloxane) " $S$ " fractions ( $\mathbf{\Delta})$ in chloroform at $30^{\circ} \mathrm{C}$. Data of ref 41.

Polymer J., Vol. 11, No. 2, 1979 with this model coincide with those estimated for fractions at finite concentrations and the same elution volume. This is because whole polymers will be diluted much more than fractionated materials in the gel-filled columns. The same effective concentrations are used here for calculations with the Yamakawa theory.

Dawkins, et al., ${ }^{41}$ have studied the GPC elution behavior of anionic polystyrenes and poly(dimethylsiloxane) fractions in chloroform at $30^{\circ} \mathrm{C}$. Here 5-mg polymer is reported to have been injected into $2-\mathrm{m} l$ eluant, so the effective polymer concentration is $0.0025 \mathrm{~g} \mathrm{~cm}^{-3}$. Figure 2 shows a common $\ln \left(V_{\mathrm{h}}\right)-V_{\mathrm{e}}$ relation for polystyrene and two different polydimethylsiloxane fractions, with hydrodynamic volumes calculated from eq 16 . The necessary parameters for this calculation are summarized in Table I. The Yamakawa theory obviously fits the data well enough. An earlier version of the simpler Rudin model was also shown to provide a single universal calibration curve. $^{12}$ It seems reasonable to conclude that the two models will both account satisfactorily for concentration effects in most GPC separations.

We now compare the calculated concentration dependence of polystyrenes of various molecular weights in a theta solvent, a good solvent (tetrahydrofuran), and a poor solvent (butanone-2). The values calculated according to eq 16 (Yamakawa) and eq 17 (Rudin) are plotted in Figure 3. The two models obviously predict the same values under theta conditions and at infinite dilution. They also yield the same $V_{\mathrm{h}} v s$. $c$ curve at low concentrations and/or for relatively low molecular weights. It is clear that the two models are equivalent to all intents and purposes at concentrations which are likely to be used in GPC analyses.

There is a difference between the two theories, however, at higher concentrations when the polymer molecular weight is high and the solvent is good. Under these circumstances, the effective hydrodynamic volume predicted by eq 12 is less than the corresponding $V_{\mathrm{h}}$ from eq 13. (Compare the plots for 498,000 molecular weight polystyrene in tetrahydrofuran in Figure 3.)

With a high molecular weight polymer in a poor solvent, however, the situation is reversed. In a poor solvent such as butanone in Figure $3, V_{\mathrm{h}}$ from the Yamakawa model is close to but slightly higher than $V_{\mathrm{h}}$ predicted from the Rudin model. The 


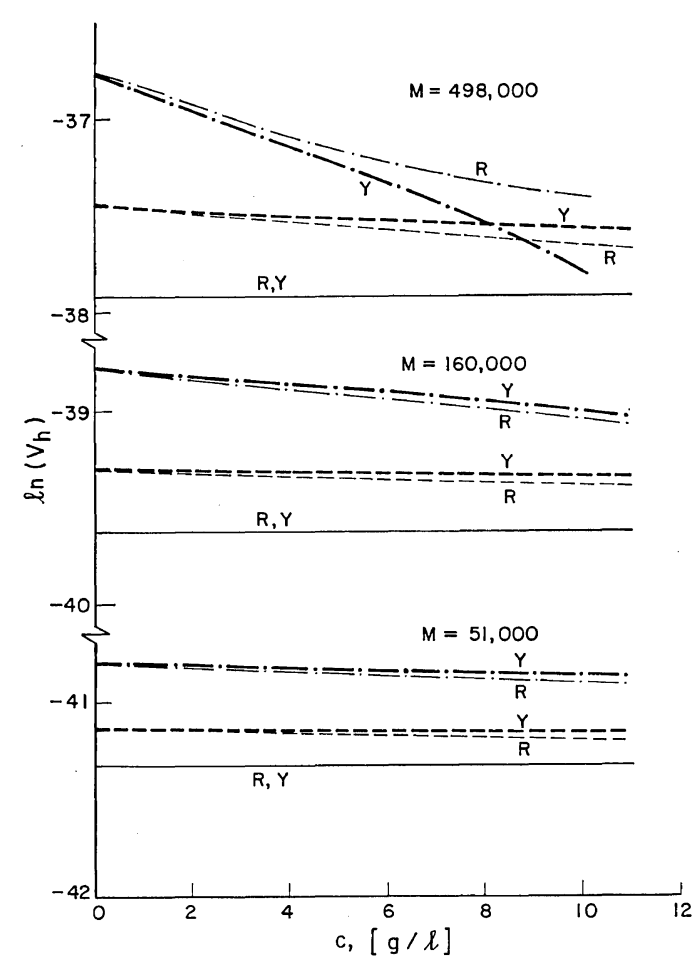

Figure 3. Plots of $\ln V_{\mathrm{h}}$ aginst concentration for polystyrenes in theta mixture (-), butanone (-----), and tetrahydrofuran (-..-) at $25^{\circ} \mathrm{C}$. Polymer molecular weights are as shown. $R$, eq $17 ; Y$, eq 16 .

absolute deviations between the two increase with concentration and increasing molecular weight.

The most surprising result of these calculations is the "crossover" predicted by the Yamakawa theory at sufficiently high molecular weight. If $M$ and $c$ are sufficiently high (compare the 498,000 molecular weight lines in Figure 3) the Yamakawa model predicts that a concentration region may exist in which $V_{\mathrm{h}}$ in a poor solvent (butanone in this case) is greater than $V_{\mathrm{h}}$ of the same polymer in a better solvent (such as tetrahydrofuran). The Rudin model does not indicate such an inversion of relative hydrodynamic volumes.

The only experimental data we could locate in this connection are the GPC results of Bleha, et al., ${ }^{5,14}$ for polystyrene solutions separated on porous glass column packings. These workers reported that elution volumes for high concentrations of high molecular weight species in a good solvent are greater than those in a poor solvent.
Mixed solvents were generally used in both cases. Some of these observations are shown in Figure 4, where it can be seen that the "cross-over" behavior was not observed for low molecular weight polymers. We do not have intrinsic viscosity data for the particular mixed solvents so that a quantitative prediction of the effect cannot be attempted.

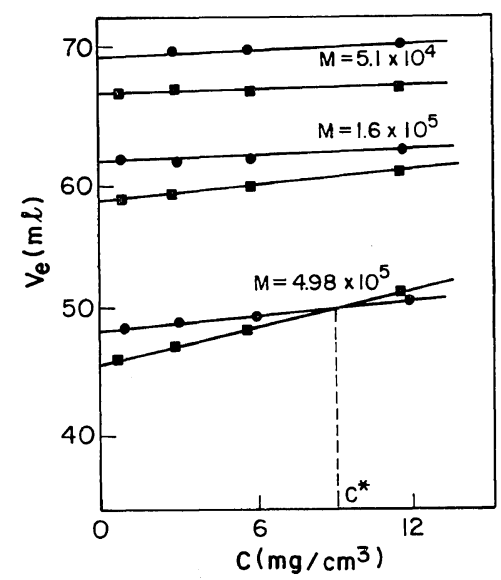

Figure 4. Plot of elution volume $\left(V_{\mathrm{e}}\right)$ against concentration for polystyrene in chloroform-methanol (90:10) (๑) and butanone-2-heptane 68.4:31.6 (घ). Data of ref 14. $c^{*}$, "cross-over" concentration.

If the behavior noted reflects the results of variations in hydrodynamic size and is not an artifact of adsorption effects, these results are consistent with the Yamakawa model. The Rudin model does not predict such a phenomenon.

The data presented here can only be obtained on inorganic column packings. Organic gels will swell differently when the solvent is changed and the same calibration curve of elution volume against hydrodynamic volume cannot be expected.

\section{Prediction of GPC Column Behavior}

The relation between elution volume, $V_{\mathrm{e}}$, and hydrodynamic volume, $V_{\mathrm{h}}$, for a particular polymer-solvent system will obviously depend on the characteristics of the GPC column set. For convenience in calculation we will assume that the calibration curve can be represented by a linear relation of the form

$$
\ln V_{\mathrm{h}}=a-b V_{\mathrm{e}}
$$

where $a$ and $b$ are constants. There is no inherent reason why such a linear relation should exist in 
GPC, but the effects of combining various pore sizes are frequently such that the calibration curve is indeed linear in the intermediate range of $V_{\mathrm{h}}$ (or $M$ ) values. In any event it is reasonable to expect that the variation of $V_{\mathrm{e}}$ with concentration for a species of given molecular weight will be reasonably linear over the range of concentration values which might be encountered in GPC. Equations 12 and 21 yield

$$
V_{\mathrm{e}}=V_{\mathrm{e} 0}+\frac{1.035}{b} Z \phi_{2}\left(\xi_{0}\right)[\eta]_{\theta} c
$$

where the elution volume at infinite dilution, $V_{\mathrm{e} 0}$, is given by

$$
V_{\mathrm{e} 0}=\frac{a+54.961}{b}-\frac{\ln (M[\eta])}{b}
$$

The constants $a$ and $b$ can be estimated by plotting $\ln (M[\eta])$ against the corresponding $V_{\mathrm{e} 0}$ values for a given column set. Equation 22 can then be used to calculate the concentration dependence of the elution volume of a polymer with given molecular weight and hence with an $[\eta]_{\theta}$ value which can be calculated from eq 3 . (It will be recalled that this procedure is valid, strictly speaking, only for sharp fractions for which the GPC separation process does not cause a significant dilution.)

The above procedures have been applied to the data of Kato and Hashimoto, ${ }^{9}$ for polystyrenes in butanone. The elution volumes for two polystyrenes in butanone and in a theta solvent were obtained by extrapolating the reported data to zero concentration. These $V_{\mathrm{e} 0}$ values were plotted against the corresponding $\ln (M[\eta])$ figures, as shown in Figure 5a. The values of the constants $a$ and $b$ were then calculated and used to calculate $V_{\mathrm{e}}$ against $c$. The resulting estimates agree well with experimental values, as shown in Figure $5 b$.

The Rudin model has previously ${ }^{13}$ been shown to account for the observations of Kato and Hashimoto as well.

It is interesting that these experimental data can be used to estimate $c_{x}$, the critical concentration at which the solvated coil dimensions are estimated to have shrunk to their unperturbed values. This can be done by extrapolating the reported elution volume lines for a good solvent and for a theta solvent. The concentration at which these elution volumes intersect is $c_{x}$. This experimental value for the $3.35 \times 10^{6}$ molecular weight polystyrene of

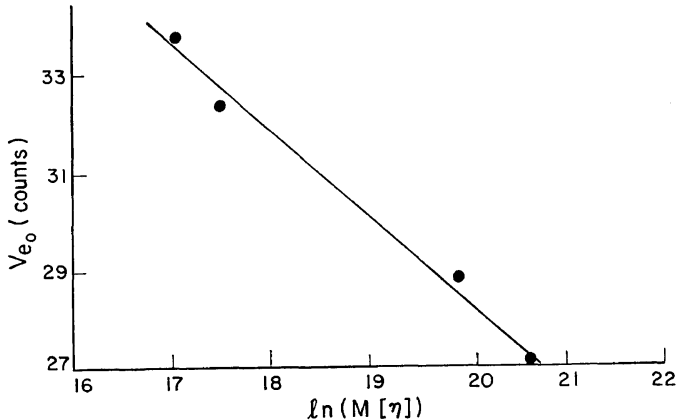

(a)

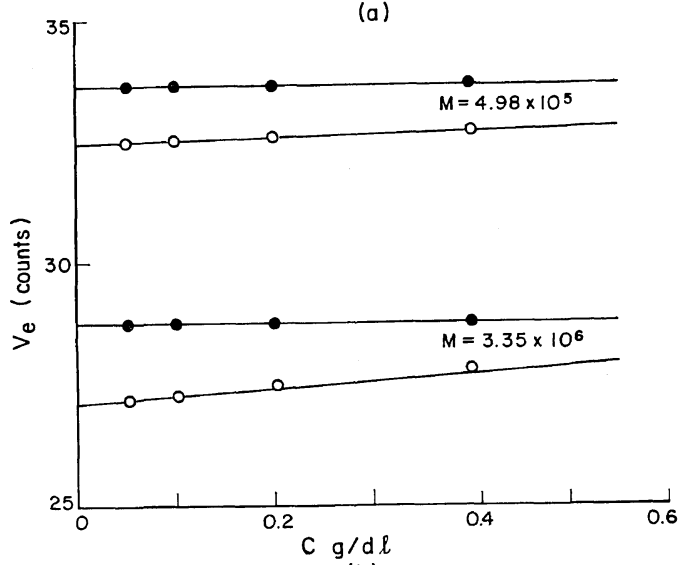

(b)

Figure 5. (a) Plot of infinite dilution elution volume $\left(V_{\mathrm{e} 0}\right)$ against $\ln ([\eta] M)$, according to eq 23. Data of ref 9. (b) Plot of $V_{\mathrm{e}}$ against concentration for polystyrene in butanone $(\bigcirc)$ and in a theta mixture $(\bullet)$ at $25^{\circ} \mathrm{C}$. Data of ref 9 . Solid line, predicted with eq 22 .

Kato and Hashimoto ${ }^{9}$ is $0.93 \mathrm{~g} / \mathrm{d} l$. Yamakawa's model (eq 14) predicts $0.95 \mathrm{~g} / \mathrm{d} l$ and Rudin's model (eq 15) yields $0.96 \mathrm{~g} / \mathrm{d} l$. The two models will yield identical $c_{x}$ values in the intermediate molecular size region.

Janca $^{16}$ has presented data for concentration effects on the elution behavior of tetrahydrofuran solutions of anionic polystyrenes on porous glass columns. This author was able to predict the elution behavior with a theory based in part on Rudin's earlier model. ${ }^{11}$ We have fitted the experimental data to the two parameter linear equation 23 to obtain an expression for the universal calibration curve in terms of $V_{\mathrm{e}}$ and $M[\eta]$. The effects of concentration for a given polystyrene follow from eq 23 of the Yamakawa model or from eq 24 for the Rudin version 


$$
V_{\mathrm{e}}=V_{\mathrm{e} 0}+\frac{1}{b} \ln \left(1+0.8137\left([\eta]-[\eta]_{\theta}\right) c\right)
$$

The results are presented graphically in Figure 6, where it can be seen that neither model can be distinguished experimentally.

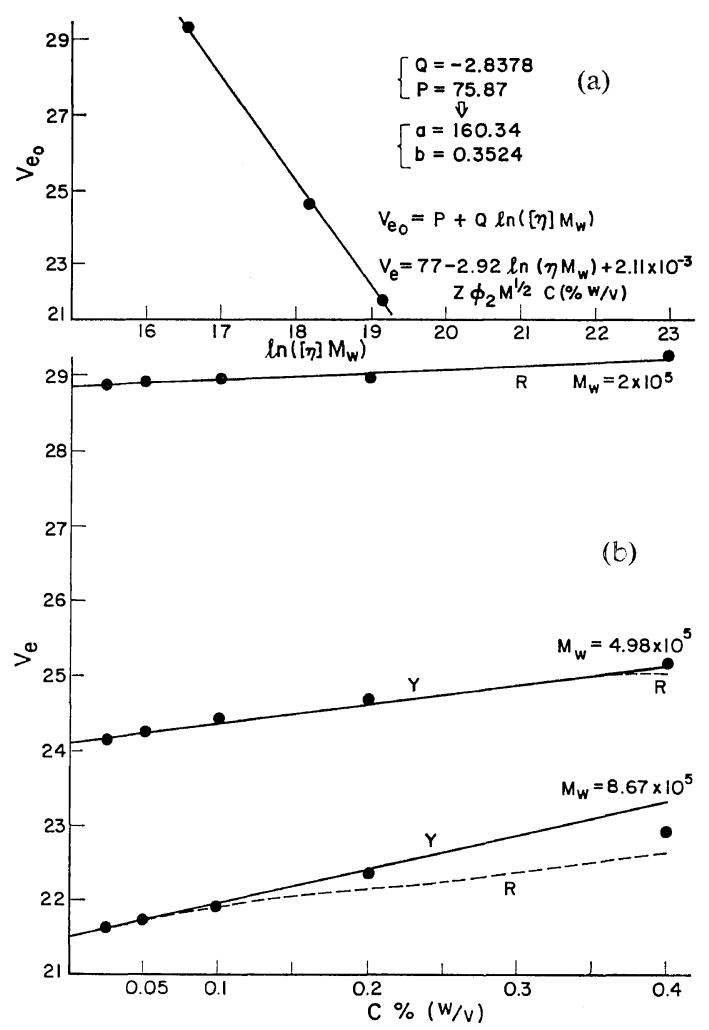

Figure 6. (a) Polystyrene in tetrahydrofuran, universal calibration curve. ${ }^{16}$ (b) Concentration dependence of elution volume. $Y$, eq $22 ; R$, eq 24 .

Much of the elution-volume/concentration data in the literature can be represented by a linear relation, for given polymer, solvent, and column set. The slope of such a relation is zero in a theta mixture and increases as the goodness of the solvent improves. Bleha, Bakos, and Berek ${ }^{14}$ have found a correlation between the slope, $K$, of such elution volume-concentration relations and the product of the second virial coefficient and polymer molecular weight.

According to eq 22 and $3, V_{\mathrm{e}}$ is a linear function of $c$ with slope proportional to $Z \phi_{2} M^{1 / 2}$ (if the universal calibration curve of $V_{\mathrm{e}}$ is linear in $\left.\ln [\eta] M\right)$. Such a plot is shown in Figure 7, where it can be

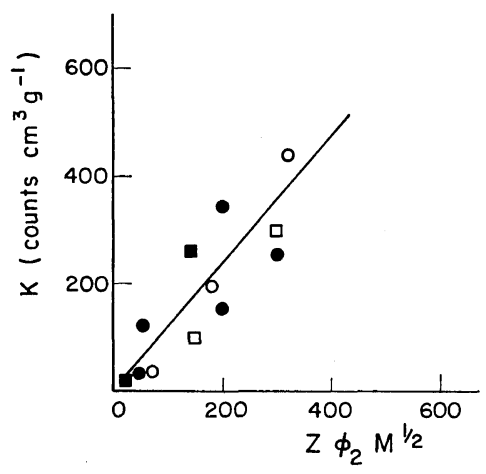

Figure 7. Plot of slope $K$ of $V_{\mathrm{e}}-c$ plot (counts $\mathrm{m} l \mathrm{~g}^{-1}$ ) against $Z \phi_{2} M^{1 / 2}$ for polystyrene in THF (๑), chloroform ( $\square$ ), butanone ( $\mathbf{\square})$, and benzene ( $\bigcirc)$. Data of ref 8 and 14. Solid line, predicted with eq 22 .

seen that the predicted linear relation is generally true.

The dependence of $V_{\mathrm{e}}$ on $c$ is not so clear-cut, inspection of eq 24 although the previous evidence of effectiveness of this equation indicates that it can also predict the $V_{\mathrm{e}}-c$ slope.

\section{DISCUSSION}

Concentration effects in GPC can be predicted and compensated for in most cases of practical interest by using either eq 12 or eq 13 . Either model can be used to establish a universal calibration from measurements of peak elution volumes of sharp distribution standard polymers measured at a single concentration. ${ }^{12}$

The theory of Yamakawa was first proposed before the advent of the GPC method and its effectiveness in this application is impressive.

As shown above, Yamakawa's calculations indicate that a concentration region may exist in which the effective hydrodynamic volume of a given high molecular weight polymer may be larger in a poorer solvent than in a thermodynamically better solvent. It is not clear how seriously this "cross-over" should be taken, since the theory was developed essentially for dilute concentrations in which the phenomenon is not expected to occur. Some GPC data from experiments with porous glass packed columns are in qualitative agreement with this "cross-over" prediction and some apparently anomalous termination rates in radical polymerizations can be rationalized by invoking this 
Effect of Solvent on Concentration Dependence of Hydrodynamic Volumes and GPC Elution Volumes

phenomenon. $^{42}$

Our conclusions regarding the utility of Yamakawa's theory differ from those of Hayashi and coworkers. $^{27}$ We have made point-to-point comparisons of calculated and measured radii of gyration whereas the cited authors were more interested in the form of the $\alpha-c$ curve.

The calculations reported here illustrate the usefulness of both the models discussed for an understanding of the operating behavior of GPC columns.

Acknowledgment. This research was supported in part by the National Research Council of Canada.

\section{REFERENCES}

1. A. Lambert, Polymer (London), 10, 213 (1969).

2. M. R. Cantow, R. S. Porter, and J. F. Johnson, J. Polym. Sci., Part B, 4, 707 (1966).

3. K. C. Berger and G.W. Schulz, Makromol. Chem., 136, 221 (1970).

4. K. A. Boni, F. A. Sliemers, and P. B. Stickney, J. Polym. Sci., Part A-2, 6, 1567 (1968).

5. D. Berek, D. Bakos, L. Soltes, and T. Bleha, $J$. Polym. Sci., Part B, 12, 277 (1974).

6. G. Meyerhoff, Ber. Bunsenges. Phys. Chem., 69, 867 (1965).

7. F. Rodriguez and O. K. Clark, Ind. Eng. Chem. Prod. Res. Dev., 5, 121 (1966).

8. D. Berek, D. Bakos, T. Bleha, and L. Soltes, Makromol. Chem., 176, 391 (1975).

9. Y. Kato and T. Hashimoto, Kobunshi Kogaku, 30, 107 (1973).

10. Y. Kato and T. Hashimoto, J. Appl. Polym. Sci., 18, 1239 (1974).

11. A. Rudin, J. Polym. Sci., Part A-1, 9, 2587 (1971).

12. A. Rudin and H. L. W. Hoegy, J. Polym. Sci., Part A-1, 10, 217 (1972).

13. A. Rudin and R. A. Wagner, J. Appl. Polym. Sci., 20, 1483 (1976).

14. T. Bleha, D. Bakos, and D. Berek, Polymer (London), 18, 897 (1977).

15. E.G. Bartick and J.F. Johnson, Polymer (London) 17, 455 (1976); Polym. Prepr., Am. Chem. Soc., Div. Polym. Chem., 17 (2), 456 (1976).

16. J. Janca, J. Chromatogr., 134, 263 (1977).
17. H. Yamakawa, J. Chem. Phys., 43, 1334 (1965).

18. P. J. Flory, "Principles of Polymer Chemistry," Cornell University Press, Ithaca, N. Y., 1953.

19. P. J. Flory and T. G. Fox, Jr., J. Am. Chem. Soc., 73, 1904 (1951).

20. C. Tanford, "Physical Chemistry of Macromolecules," John Wiley, N. Y., 1961, p 168.

21. J. Oth and V. Desreux, Bull. Soc. Chim. Belg., 18, 830 (1954).

22. S. N. Chinai, P. C. Scherer, C. W. Bondurant, and D. W. Levi, J. Polym. Sci., 22, 527 (1956).

23. E. F. Casassa and G. C. Berry, J. Polym. Sci., Macromol. Rev., 4, 1 (1970).

24. T. Kawai and K. Saito, J. Polym. Sci., 26, 213 (1957).

25. M. Fixman, J. Chem. Phys., 33, 370 (1960); Ann. N. Y. Acad. Sci., 89, 657 (1961); J. Polym. Sci., 47, 91 (1960).

26. H. Yamakawa, Pure Appl. Chem., 31, 179 (1972).

27. H. Hayashi, F. Hamada, and A. Nakajima, Macromolecules, 7, 959 (1974).

28. T. B. Grimley, Trans. Faraday Soc., 57, 1974 (1961).

29. R. Simha and J. L. Zakin, J. Chem. Phys., 33, 1791 (1960).

30. P. Outer, C. I. Carr, and B. H. Zimm, J. Chem. Phys., 18, 830 (1950).

31. G. C. Berry, J. Chem. Phys., 44, 4550 (1966).

32. R. G. Kirste and G. Wild, Makromol. Chem., 121, 174 (1966).

33. H. Hayashi, F. Hamada, and A. Nakajima, Macromolecules, 9, 543 (1976).

34. T. A. Orofino and F. Wenger, J. Phys. Chem., 67, 566 (1963).

35. G. V. Schulz and A. Haug, Z. Phys. Chem. (Frankfurt), N. F., 34, 328 (1962).

36. W. B. Smith and A. Kollmansberger, J. Phys. Chem., 69, 4157 (1965).

37. J. Cazes and D. R. Gaskell, Sep. Sci., 2, 421 (1967).

38. J. C. Hendrickson and J. C. Moore, J. Polym. Sci., Part A-1, 4, 167 (1966).

39. Z. Grubisic, P. Rempp, and H. Benoit, J. Polym. Sci., Part B, 5, 753 (1967).

40. Z. Grubisic and H. Benoit, C. R. Acad. Sci. (Paris), Ser. C, 266, 1275 (1968).

41. J. V. Dawkins, J. W. Maddock, and D. Coupe, J. Polym. Sci., Part A-2, 8, 1803 (1970).

42. H. Kh. Mahabadi and A. Rudin, J. Polym. Sci., Chem. Ed., in press. 